\title{
Factores que influyen en la percepción de los profesores de los beneficios instruccionales de los medios educativos digitales
}

\author{
Factors affecting school teachers' perceptions of the instructional benefits of educational digital \\ media
}

\author{
Badia, Antoni; Meneses, Julio; Fàbregues Sergi \& Sigalés, Carles \\ Universitat Oberta de Catalunya
}

\begin{abstract}
Resumen
Este estudio pretende identificar los factores que influyen en las percepciones de los profesores sobre los beneficios de los medios educativos digitales para la enseñanza y el aprendizaje. Varios estudios relevantes han indicado que cuando los profesores creen que los medios educativos digitales son valiosos para la enseñanza y el aprendizaje, son más propensos a incorporarlos en su práctica docente. Se han considerado tres conjuntos de factores como variables independientes: las características de la escuela y del profesor, las características tecnológicas de la escuela, y las características tecnológicas del profesor. Se recogieron datos de 702 profesores de unas 356 escuelas de educación primaria y secundaria en España con un cuestionario. Mediante análisis de correlación múltiple y de regresión jerárquica examinamos la relación entre las variables independientes y las percepciones de los profesores. Los resultados muestran una fuerte relación entre estas percepciones y las características tecnológicas del profesor. Los factores más predictivos son el área de enseñanza, la alfabetización digital, la formación en tecnología educativa y la frecuencia de acceso a Internet, dentro y fuera de la escuela. Por último, sugerimos que la integración de los medios educativos digitales en el aula no es un objetivo aislado que debe ser alcanzado por separado de los objetivos pedagógicos, sino que es un objetivo totalmente interrelacionados con las finalidades instruccionales de los profesores.
\end{abstract}

Palabras clave: Medios educativos digitales, Aprendizaje asistido por ordenador, competencias del profesor, creencias del profesor, escuelas

\begin{abstract}
This study aims to identify the factors that affect primary and secondary school teachers' perceptions of the potential benefits of digital media for teaching and learning in schools. Various studies have shown that when teachers believe that the use of digital media is valuable for instruction and learning, they are more likely to incorporate their use into teaching practice. Three sets of factors were used as independent variables: the general profile of the school and the teacher, the technological profile of the school, and the technological profile of the teacher. This study used survey data gathered from 702 teachers at 356 primary and secondary schools in Spain. Correlation and hierarchical regression analyses were performed to examine the relationship between the independent variables and teachers' perceptions. The results show a strong relationship between these perceptions and the technological profile of teachers. The factors that best predict the dependent variable are the area of study, digital literacy, ICT training, and the frequency of Internet access, both inside and outside the school. Finally, we suggest that the integration of digital media in the classroom is not an isolated objective to be achieved separately from pedagogical goals, but is closely interrelated with teachers' educational aims.
\end{abstract}

Fecha de recepción 16 de Diciembre 2014

Fecha de aprobación 6 de Julio de 2015

Fecha de publicación

10 de Octubre de 2015

Reception Date 2014 December 16

Approval Date 2015 July 6

Publication Date: 2015 October 10

Keywords: Educational digital media, computer assisted learning, teacher competencies, teacher beliefs, schools 
La identificación de los factores que pueden influir en el uso de los medios educativos digitales en la enseñanza y el aprendizaje se ha convertido en la última década en un tema central de estudio en el campo del aprendizaje basado en la tecnología (Badia, Meneses y Sigalés, 2013; Tondeur, Valcke, y Van Braak, 2008; Van Braak, 2001; Wastiau, Blamire, Kearney, Quittre, Van de Gaer, y Monseur, 2013).

Uno de los factores relevantes más citados es la percepción del profesor de los beneficios instruccionales de los medios educativos digitales (Inan y Lowther, 2010; Knezek, Christensen, y Fluke, 2003; Van Braak, Tondeur y Valcke, 2004). Según Petko (2012, p. 1355), la efectividad de la tecnología en el aprendizaje es "la creencia de que el aprendizaje del estudiante mejora con la ayuda de la tecnología digital [...]. El uso de los medios digitales puede mejorar la calidad de la enseñanza, los resultados del aprendizaje, el interés y la creatividad, el trabajo colaborativo y las estrategias de aprendizaje de los alumnos".

La relación entre la percepción del profesor de los beneficios de los medios educativos digitales y la frecuencia de uso en las aulas ha sido demostrada por varias investigaciones. Van Braak, Tondeur y Valcke (2004) incluyeron este aspecto dentro de una variable más amplia que medía las actitudes de los profesores hacia los ordenadores en la educación. Algunos ítems que estos autores utilizaron fueron: "Aumenta el nivel de creatividad de los alumnos", "ayuda a los alumnos a lograr una mejor escritura de texto" o "cuando la tecnología es utilizada como herramienta de aprendizaje, aumenta la motivación de los estudiantes". Los resultados mostraron la existencia de cinco variables que afectan la frecuencia de uso de los ordenadores en el aula: Las actitudes de los profesores hacia los ordenadores en la educación, la innovación tecnológica, la intensidad de uso del ordenador, la formación en ordenadores y el género. La variable que medía las actitudes de los profesores hacia los ordenadores en la educación estaba muy influida por las actitudes generales de los profesores hacia los ordenadores.

Inan y Lowther (2010) también incluyeron la percepción de los beneficios potenciales de los medios educativos digitales en su análisis, que consistió en examinar la influencia de las características individuales de los profesores y sus percepciones de los factores contextuales en la integración de la tecnología en las aulas. Los resultados mostraron que varios factores tuvieron una influencia significativa: el grado de competencia en el uso del ordenador, el nivel global de soporte, el soporte técnico, la disponibilidad de ordenadores, y las creencias de los profesores sobre la tecnología. Este último aspecto fue definido como la percepción de los profesores sobre la influencia de la tecnología en la enseñanza y aprendizaje.

Wastiau et al. (2013) también señalaron que la percepción del profesor de la influencia de los medios educativos digitales en la motivación y el rendimiento de los alumnos, y en los procesos y los resultados de aprendizaje, es una variable importante para comprender el uso de la tecnología en las aulas. Otros factores influentes son el tipo de liderazgo y la estrategia de integración de la tecnología, la infraestructura tecnológica y el acceso a la tecnología, y la auto-percepción de la competencia digital de profesores y alumnos.

La relación entre los beneficios percibidos y la frecuencia de uso de la tecnología y los medios educativos digitales en el aprendizaje ha sido confirmada por una línea de investigación que han contribuido a la construcción del modelo explicativo denominado "will, skill, tool", propuesto inicialmente por Christensen y Knezek (2001) y continuado por estudios posteriores (Christensen y Knezek, 2008; Knezek, Christensen y Fluke, 2003; Morales, 2006; Petko, 2012). Tres grupos de factores, denominados voluntad de uso, competencia en el uso y disponibilidad de tecnología, explican gran parte de la varianza de la frecuencia de uso de la tecnología y los medios educativos digitales en el aula. El factor voluntad de uso 
está basado en la efectividad percibida de la tecnología digital, y abarca el conjunto de creencias del profesor sobre la utilidad de la tecnología. El factor competencia de uso incluye las competencias de los profesores para integrar la tecnología digital en la enseñanza, y el factor disponibilidad de la tecnología abarca las condiciones de acceso y las características de la infraestructura tecnológica.

Esta relación también se ha evidenciado en estudios comparativos, entre profesores que integran y que no integran la tecnología en sus aulas. De acuerdo con Mueller, Wood, Willoughby, Ross y Specht (2008) existen dos grupos de variables discriminantes. El primer grupo está relacionado con la experiencia previa de los profesores con la tecnología y los ordenadores, e incluye haber experimentado prácticas docentes positivas con ordenadores, sentir confort y entusiasmo trabajando con ordenadores, haber recibido formación, y haber recibido la ayuda de otros. El segundo grupo de factores tiene que ver con las actitudes de los profesores frente la tecnología, y comprende la valoración de la tecnología como una herramienta instruccional adecuada, la percepción de utilidad y motivación para usar los medios educativos digitales, y la creencia que la actividad de enseñanza del profesor puede influir en los estudiantes.

La relación entre los beneficios potenciales de Internet y los medios educativos digitales y su uso en el aula también se constata en colectivos de profesores muy formados en el uso de las TIC que enseñan en aulas altamente dotadas de tecnología. Badia, Meneses y Sigalés (2013) señalaron que uno de los factores que más influyó en el uso educativo de Internet y los medios educativos digitales en este tipo de profesores y aulas fue la percepción del profesor de la utilidad de las tecnologías para el aprendizaje. Este factor midió la percepción de los profesores en relación con el grado en que las tecnologías mejoran la calidad del aprendizaje, los resultados académicos y la consecución de los objetivos de aprendizaje.

Mientras que los estudios anteriores se han centrado en constatar la relación entre las creencias del profesor que la tecnología produce beneficios en el aprendizaje y el uso de la tecnología en el aula, se han llevado a cabo escasas investigaciones sobre cuáles son los principales factores que influyen en la percepción de los profesores sobre la utilidad de la tecnología en el aprendizaje.

Hasta la fecha, únicamente Perrotta (2013) ha estudiado cómo algunos aspectos del sistema educativo, tales como las características de la institución educativa, del aula y del profesor, pueden influir en los beneficios percibidos del uso de la tecnología por parte de los docentes. En su estudio se valoró varios beneficios educativos de la tecnología por parte de los profesores y alumnos, tales como un mayor acceso a recursos y contenidos de aprendizaje, una mayor motivación y atención al aprender, y una mayor posibilidad que los alumnos aprendan de una manera más activa e independiente. Los resultados evidenciaron la percepción de los profesores está más influenciada por características institucionales de la escuela que por características individuales del profesor. En consecuencia, Perrotta sostuvo que algunos factores relativos a las condiciones socioeconómicas e institucionales son más influyentes que las características del aula y las características individuales de los profesores. Estos factores son la naturaleza de las condiciones de la institución educativa, las características de los contextos reales en donde trabajan los profesores, y las expectativas culturales que rodean cada área curricular.

Nuestra investigación pretende ampliar el conocimiento actualmente disponible sobre esta temática mediante el desarrollo de un modelo explicativo de los factores que afectan la percepción de la efectividad de los medios educativos digitales en las prácticas docentes de profesores de educación primaria y educación secundaria. Para conseguir esta finalidad, en primer lugar nos proponemos identificar qué grupos de factores ejercen efectos significativos más positivos. Siguiendo a Tondeur (2007), a nivel estrictamente analítico agruparemos los factores en tres 
categorías, que denominamos: a) Características estructurales de la escuela y características individuales del profesor; b) Características tecnológicas de la escuela; y c) Características tecnológicas del profesor. En segundo lugar, nos proponemos identificar cuáles son los factores específicos que mayor influencia ejercen en la percepción de los profesores de los beneficios potenciales de la tecnología.

\section{Método}

\section{Recogida de datos}

Los datos fueron recogidos en el marco de una investigación más amplia llevada a cabo durante los años 2007-2009, que supuso un análisis de la integración de Internet en la educación escolar española (Sigalés, Mominó, Meneses y Badia, 2009). En una primera fase se formó a un equipo de entrevistadores para la recogida de datos. En paralelo, se contactó con las escuelas mediante una carta, solicitando la participación del centro, y una semana después se contactó telefónicamente con los directores para concertar una primera visita. La segunda fase consistió en la recogida de datos en los centros. Se utilizaron dos cuestionarios escritos, uno para los directores y otro para los profesores, que participaron de forma voluntaria y anónima. Para completar la recogida de datos se realizaron entre 3 y 4 visitas a cada centro. Finalmente, los datos fueron depurados e introducidos en una base de datos.

\section{Participantes}

Participaron una muestra de 356 centros educativos de educación primaria y secundaria obligatoria en España. Dentro de cada centro, los directores y los docentes seleccionaron al azar un aula perteneciente a un curso de final de ciclo (de alumnos de 11-12 años en educación primaria, y de alumnos de 15-16 años en educación secundaria). Con la ayuda y orientación de los miembros del equipo de investigación, la totalidad de directores de los centros y los profesores que imparten docencia en dicha aula respondieron al cuestionario. La muestra final del estudio fue de 356 directores y 702 docentes. El 59\% de los participantes fueron mujeres y el $41 \%$ hombres, con una edad media de 41 años.

\section{Instrumentos de medida}

\section{Características estructurales de la escuela y características individuales del profesor}

Se solicitó que los profesores aportaran información sobre su edad, sexo y principal área curricular. También se pidió que aportaran información sobre varios aspectos de su institución escolar: nivel educativo (educación primaria o educación secundaria), tipo de titularidad (pública o privada), y población de la ciudad en la que se encuentra la institución escolar.

\section{Características tecnológicas de la escuela}

Se pidió a los directores de las instituciones educativas que aportaran información sobre la política del centro sobre la integración tecnológica, la dotación en infraestructura tecnológica, el porcentaje de ordenadores disponibles por alumno, y la disponibilidad de apoyo al profesorado para el uso de la tecnología y los medios educativos digitales.

Para evaluar las políticas del centro respecto la tecnología se presentó a los profesores una escala de seis ítems de respuesta dicotómica (sí/no) sobre la enseñanza y la gestión. Los tres ítems sobre las políticas de enseñanza sirvieron para recoger información sobre la existencia de medidas para promover cambios en las metas educativas y los planes de estudio, para fomentar nuevos métodos de enseñanza, y para mejorar las prácticas de evaluación de los aprendizajes. Los tres ítems de políticas de gestión recabaron información sobre la existencia de medidas para promover el uso de la tecnología por parte de los estudiantes y sus familias fuera de la escuela, de medidas de incentivos y recompensas a los profesores para promover el uso de la tecnología en sus prácticas pedagógicas, y de medidas para promover la competencia docente en el uso educativo de la tecnología. Dado su carácter no métrico, fue utilizado un PCA con correlación policórica que generó una estructura de dos componentes $(\mathrm{KMO}=.714$ y una prueba de 
Barlett significativa, $\mathrm{p}=.000)$. La varianza total (65.32\%) se distribuyó entre el $48.35 \%$ referida a las políticas de enseñanza y el $16.97 \%$ referida a las políticas de gestión. La solución rotada (oblimin directo) proporcionó componentes de cargas que van desde .763 hasta .905, y desde .726 hasta .745, respectivamente. Ambos componentes mostraron una fiabilidad aceptable, con unas $\alpha$ de Cronbach de .901 y .790, respectivamente.

Para analizar la infraestructura tecnológica del centro educativo se presentó una lista de cuatro ítems de respuesta dicotómica (sí/no) sobre la infraestructura tecnológica disponible en los centros educativos: el mantenimiento de la página web de la escuela, el acceso a Internet de banda ancha, la existencia de WIFI, y la disponibilidad de una intranet escolar. El análisis de componentes principales mostró una estructura de un factor $(\mathrm{KMO}=.688$ y una prueba de Bartlett significativa, $\mathrm{p}=.000$ ), con cargas factoriales que oscilan entre .594 y .827. Esta solución representó el 50.63 \% de la varianza total $\mathrm{y}$ mostró una fiabilidad aceptable, con un $\alpha$ de Cronbach de .675.

\section{Características tecnológicas del profesor}

Los profesores aportaron información sobre el grado de alfabetización digital, nivel de formación, y frecuencia de acceso a Internet dentro y fuera de la escuela.

Para evaluar la competencia digital los profesores informaron sobre su grado de habilidad en seis tareas tecnológicas típicas de Internet: navegar por Internet, descargar un archivo, enviar un correo electrónico, utilizar aplicaciones de mensajería instantánea, publicar información en Internet y desarrollar páginas web. Existían cuatro tipos de respuestas que correspondían a cuatro niveles de dominio, que van desde (1) "No sé lo que es o qué significa" a (4) "Puedo hacerlo por mí mismo". El análisis de componentes principales mostró una solución aceptable de un componente $(\mathrm{KMO}=.843$ y una prueba de Bartlett significativa, $\mathrm{p}=.000$ ), con saturaciones en los componentes que oscilan desde .771 a .903. Esta solución representó el 72.33 \% de la varianza total y mostró un $\alpha$ de Cronbach de .921 .

El nivel de formación tecnológica de los profesores se midió mediante un ítem que recoge información tanto de la cantidad como de la calidad de los cursos de formación recibidos por los profesores en los últimos tres años. Las tres posibles respuestas fueron: "No se recibió ningún curso de formación”, "Se recibió un curso de formación pero fue poco útil", y "Se recibió un curso de formación y fue útil”.

Por último, para medir la frecuencia de acceso a Internet dentro del centro educativo (es decir, desde la sala de profesores, la biblioteca, o la sala de ordenadores) y fuera del centro y en horario no escolar (es decir, desde el hogar, una biblioteca pública, o un cibercafé, entre otros) se utilizaron dos ítems con cuatro niveles de respuesta, que oscilan entre (1) "nunca o casi nunca” y (4) "todos los días".

\section{Beneficios percibidos de los medios educativos digitales}

Los profesores respondieron a cinco ítems que se refieren a los beneficios que los medios educativos digitales aportan a sus prácticas de enseñanza. Estos ítems comprenden tanto aspectos de desarrollo curricular como aspectos relativos a las actividades de enseñanza y aprendizaje de los alumnos. Los ítems son: 1) La comunicación y la colaboración con mis alumnos desarrollada mediante la tecnología digital es útil para mi práctica docente: 2) Los recursos educativos disponibles en Internet satisfacen las necesidades de mi área curricular; 3) La tecnología mejora la calidad de las experiencias de aprendizaje de mis alumnos; 4) La tecnología contribuye a alcanzar los objetivos educativos de mis alumnos, y 5) Los recursos educativos diseñados con tecnología son adecuados para mi enseñanza. Los profesores valoraron cada ítem mediante una escala de cinco posibles respuestas, que oscilan entre (1) "muy en desacuerdo" a (5) "totalmente de acuerdo". El análisis factorial exploratorio utilizando el análisis de componentes principales (PCA) mostró una solución de un 
solo componente $(\mathrm{KMO}=.857$ y una prueba de Bartlett significativa, $p=.000$ ), con saturaciones en los componentes que van desde .770 hasta .847. Esta solución representó el $64.50 \%$ de la varianza total con un $\alpha$ de Cronbach de .861 .

\section{Análisis de datos}

El análisis de los datos se realizó con el programa SPSS 21. En primer lugar, se comprobó que se cumplieran todos los supuestos para poder realizar las pruebas estadísticas previstas. En segundo lugar, se correlacionaron todas las variables entre sí para obtener un primer índice de validez para las hipótesis, así como también para poner a prueba el grado de independencia de los factores influyentes.

En tercer lugar, con el fin de contrastar las relaciones bivariadas y estimar la contribución relativa de las variables explicativas, se realizó una regresión jerárquica de tres pasos. Este tipo de regresión permite examinar la influencia de las variables independientes de un modo secuencial, de manera que el investigador puede determinar, paso a paso, en qué medida la inclusión de nuevas variables contribuye a mejorar la varianza explicada respecto a los modelos anteriores. La elección de este tipo de regresión se hizo en función de los objetivos de esta investigación. Además de mostrar la influencia de cada variable independiente, también nos proponemos constatar la influencia de cada uno de los tres grupos de variables. El primer modelo muestra la influencia de las variables de control, referidas a las características estructurales de la escuela y las características individuales del profesor. El segundo modelo revela la influencia del anterior grupo de variables junto a las variables relativas a las características tecnológicas de la escuela. Finalmente, el tercer modelo muestra la influencia de los dos anteriores grupos de variables junto con las características tecnológicas del profesor.

\section{Resultados}

\section{Datos descriptivos y correlacionales}

La Tabla 1 muestra las estadísticas descriptivas y las correlaciones entre todas las variables.

Tabla 1 - Medias, desviaciones estándar y correlaciones entre las variables observadas

\begin{tabular}{|c|c|c|c|c|c|c|c|c|c|c|c|c|c|c|c|c|c|c|}
\hline & & $\mathrm{M}$ & SD & 1 & 2 & 3 & 4 & 5 & 6 & 7 & 8 & 9 & 10 & 11 & 12 & 13 & 14 & 15 \\
\hline 1 & Percepción de los beneficios ${ }^{e}$ & 2.49 & 0.64 & & & & & & & & & & & & & & & \\
\hline 2 & Alfabetización digital ${ }^{\mathrm{f}}$ & 2.35 & 0.53 & $.28^{\mathrm{d}}$ & & & & & & & & & & & & & & \\
\hline 3 & Formación uso educativo tecnología g & 1.12 & 0.88 & $.26^{\mathrm{d}}$ & $.23^{\mathrm{d}}$ & - & & & & & & & & & & & & \\
\hline 4 & Acceso a Internet en la escuela ${ }^{\mathrm{h}}$ & 1.97 & 1.06 & $.25^{\mathrm{d}}$ & $.33^{\mathrm{d}}$ & $.24^{\mathrm{d}}$ & - & & & & & & & & & & & \\
\hline 5 & Acceso a Internet fuera la escuela ${ }^{i}$ & 2.13 & 1.08 & $.20^{\mathrm{d}}$ & $.49^{\mathrm{d}}$ & $.14^{\mathrm{d}}$ & $.30^{\mathrm{d}}$ & - & & & & & & & & & & \\
\hline 6 & Política sobre enseñanza con tecnología & 0.33 & 0.35 & $.07^{\mathrm{b}}$ & .03 & .02 & $.09^{\mathrm{c}}$ & -.13 & - & & & & & & & & & \\
\hline 7 & Política sobre gestión con tecnología & 0.48 & 0.29 & .05 & .02 & .06 & .04 & .00 & $.36^{\mathrm{d}}$ & - & & & & & & & & \\
\hline 8 & Infraestructura tecnológica & 0.61 & 0.27 & $.06^{\mathrm{a}}$ & .02 & $.11^{\mathrm{c}}$ & .05 & -.03 & $.07^{\mathrm{a}}$ & $.22^{\mathrm{d}}$ & - & & & & & & & \\
\hline 9 & Ratio ordenadores alumno & 0.45 & 1.02 & -.02 & -.04 & $.09^{\mathrm{c}}$ & $.13^{\mathrm{d}}$ & -.05 & .04 & .05 & -.04 & - & & & & & & \\
\hline 10 & Apoyo al uso de la tecnología ${ }^{j}$ & 0.63 & 0.48 & -.09 & $.06^{\mathrm{a}}$ & $.10^{\mathrm{c}}$ & $.12^{\mathrm{b}}$ & .02 & $.08^{\mathrm{b}}$ & $.14^{\mathrm{d}}$ & $.11^{\mathrm{c}}$ & .02 & - & & & & & \\
\hline 11 & Edad & 41.09 & 9.86 & -.03 & $-.33^{\mathrm{d}}$ & .05 & $-.06^{\mathrm{a}}$ & $-.20^{\mathrm{d}}$ & -.02 & .05 & $.13^{\mathrm{d}}$ & -.00 & .03 & - & & & & \\
\hline 12 & Género ${ }^{k}$ & 0.41 & 0.49 & $.11^{\mathrm{c}}$ & $.11^{\mathrm{c}}$ & .05 & $.11^{\mathrm{c}}$ & $.07^{\mathrm{b}}$ & -.03 & .02 & .03 & $-.06^{\mathrm{a}}$ & -.03 & $.13^{\mathrm{d}}$ & - & & & \\
\hline 13 & Área curricular ${ }^{1}$ & 2.94 & 2.01 & $.09^{\mathrm{c}}$ & $.16^{\mathrm{d}}$ & .00 & $.13^{\mathrm{d}}$ & $.10^{\mathrm{c}}$ & .04 & .01 & -.02 & .03 & .01 & -.04 & $.11^{\mathrm{c}}$ & - & & \\
\hline 14 & Nivel educativo $^{\mathrm{m}}$ & 0.20 & 0.40 & .04 & -.01 & $-.08^{b}$ & -.04 & -.01 & $.11^{\mathrm{c}}$ & .00 & $.13^{\mathrm{d}}$ & $-.10^{c}$ & -.00 & -.05 & $.12^{\mathrm{d}}$ & $.07^{\mathrm{b}}$ & - & \\
\hline 15 & Titularidad $^{\mathrm{n}}$ & 0.37 & 0.48 & -.01 & -.02 & $-.11^{\mathrm{c}}$ & $-.13^{\mathrm{d}}$ & -.00 & $.10^{\mathrm{c}}$ & .05 & $.20^{\mathrm{d}}$ & $-.15^{\mathrm{d}}$ & .00 & -.04 & $.14^{\mathrm{d}}$ & .03 & $.61^{\mathrm{d}}$ & - \\
\hline 16 & Población ciudad ${ }^{\circ}$ & 1.82 & 1.37 & -.00 & -.02 & -.02 & $-.12^{\mathrm{d}}$ & .02 & .01 & $.10^{c}$ & $.11^{\mathrm{c}}$ & $-.14^{\mathrm{d}}$ & $.07^{\mathrm{b}}$ & $.06^{\mathrm{a}}$ & $.08^{\mathrm{b}}$ & -.01 & $.19^{\mathrm{d}}$ & $.33^{\mathrm{d}}$ \\
\hline
\end{tabular}

${ }^{e} 0=$ "muy en desacuerdo", $4=$ muy de acuerdo; ${ }^{f} 0=$ No sé lo qué es o qué significa”, $3=$ Puedo hacerlo por mi mismo; ${ }^{g} 0=$ Ninguna, $1=$ Sí y fue poco útil, $2=$ Sí y fue muy útil; ${ }^{h} 0=$ Nunca o casi nunca, $3=$ Diariamente; ${ }^{i} 0=$ Nunca o casi nunca, $3=$ Diariamente; ${ }^{j}$ $0=$ No, $1=$ Sí; ${ }^{k} 0=$ Mujer, $1=$ Hombre; ${ }^{l} 0=$ Lengua española, $1=$ Lengua cooficial en cada comunidad autónoma, $2=$ Lengua inglesa 3=Matemáticas, 4=Ciencias sociales, 5=Ciencias naturales, 6=Educación visual y plástica, 7=Tecnología; m $0=$ =ducación primaria, $1=$ Educación secundaria; ${ }^{n} 0=$ Pública, $1=$ Privada; ${ }^{\circ} 0=$ Menos que 10.000, 4=500.001 o más.

Únicamente algunas variables tienen una correlación positiva significativa con los beneficios percibidos de los medios educativos digitales. Las relaciones más intensas se producen en las variables relacionadas con las características tecnológicas del profesor, tales como el grado de alfabetización digital ( $\mathrm{r}=.28$, $\mathrm{p}<.001$ ), la formación recibida y la valoración de su grado de utilidad $(\mathrm{r}=.26, \mathrm{p}<.001)$, la frecuencia de acceso a Internet en el centro 
Badia, Antoni; Meneses, Julio; Fàbregues Sergi \& Sigalés, Carles (2015). Factores que influyen en la percepción de los profesores de los beneficios instruccionales de los medios educativos digitales. RELIEVE, v. 21(2), art. 1. DOI: $\underline{\text { http://dx.doi.org/10.7203/relieve.21.1.7204 }}$

escolar $(\mathrm{r}=.25, \mathrm{p}<.001)$ y la frecuencia de acceso a Internet fuera del centro escolar $(\mathrm{r}=.20, \mathrm{p}<.001)$. Otras variables que también presentan una relación positiva y significativa, aunque más débil, son el género $(\mathrm{r}=.11, \mathrm{p}$ $<.01$ ), el área curricular ( $\mathrm{r}=.09, \mathrm{p}<.01)$, y la política de enseñanza con tecnología $(r=.07, \mathrm{p}$ $<.05$ ). Por último, la variable infraestructura tecnológica también muestra una correlación positiva, aunque únicamente con una cierta tendencia a la significatividad $(r=.06, \mathrm{p}<.10)$.

\section{Análisis de regresión jerárquica}

La tabla 2 muestra los resultados de los tres modelos. Con el fin de responder a los dos objetivos de la investigación, en primer lugar comentaremos la influencia de cada categoría de variables, y seguidamente especificaremos los efectos de cada variable independiente en función de cada modelo.

Tabla 2 - Modelos de regresión jerárquica de los factores que influyen en la percepción de los profesores de los beneficios instruccionales de los medios educativos digitales

\begin{tabular}{|c|c|c|c|c|c|c|c|c|c|}
\hline \multirow[t]{2}{*}{. } & \multicolumn{3}{|c|}{ Modelo 1} & \multicolumn{3}{|c|}{ Modelo 2} & \multicolumn{3}{|c|}{ Modelo 3} \\
\hline & B (S.E.) & Beta & $\mathbf{t}$ & B (S.E.) & Beta & $\mathrm{t}$ & B (S.E.) & Beta & t \\
\hline Constante & $2.404(0.136)$ & - & $17.674^{\mathrm{d}}$ & $2.277(0.146)$ & - & $15.594^{\mathrm{d}}$ & $1.534(0.210)$ & - & $7.302^{\mathrm{d}}$ \\
\hline \multicolumn{10}{|l|}{ Nivel educativo } \\
\hline Primaria & - & - & - & - & - & - & - & - & - \\
\hline Secundaria & $0.66(0.081)$ & .040 & 0.813 & $0.052(0.081)$ & .032 & 0.648 & $0.035(0.076)$ & .021 & 0.459 \\
\hline \multicolumn{10}{|l|}{ Titularidad } \\
\hline Pública & - & - & - & - & - & - & - & - & - \\
\hline Privada & $-0.085(0.071)$ & -.063 & -1.206 & $-0.124(0.072)$ & -.092 & $-1.731^{\mathrm{a}}$ & $-0.037(0.069)$ & -.027 & -0.533 \\
\hline \multicolumn{10}{|l|}{ Población ciudad } \\
\hline Menos que 10.000 & - & - & - & - & - & - & - & - & - \\
\hline $10.001-50.000$ & $0.023(0.077)$ & .016 & 0.302 & $0.060(0.078)$ & .041 & 0.778 & $0.053(0.074)$ & .036 & 0.727 \\
\hline $50.001-100.000$ & $0.091(0.099)$ & .041 & 0.920 & $0.109(0.101)$ & .049 & 1.080 & $0.130(0.095)$ & .059 & 1.370 \\
\hline $100.001-500.000$ & $0.017(0.076)$ & .012 & 0.218 & $0.022(0.076)$ & .015 & 0.286 & $0.033(0.072)$ & .023 & 0.454 \\
\hline 500.001 o más & $-0.025(0.096)$ & -.013 & -0.257 & $-0.004(0.098)$ & -.002 & -0.042 & $-0.005(0.092)$ & -.003 & -0.059 \\
\hline Edad & $-0.004(0.003)$ & -.056 & -1.375 & $-0.005(0.003)$ & -.068 & $-1.639^{\mathrm{a}}$ & $0.000(0.003)$ & -.006 & -0.131 \\
\hline \multicolumn{10}{|l|}{ Género } \\
\hline Mujer & - & - & - & - & - & - & - & - & - \\
\hline Hombre & $0.144(0.054)$ & .110 & $2.664^{\mathrm{C}}$ & $0.146(0.054)$ & .111 & $2.689^{c}$ & $0.077(0.052)$ & .059 & 1.479 \\
\hline \multicolumn{10}{|l|}{ Área curricular } \\
\hline Lengua española & - & - & - & - & - & - & - & - & - \\
\hline Lengua CCAA & $0.121(0.118)$ & .046 & 1.026 & $0.120(0.118)$ & .046 & 1.022 & $0.067(0.112)$ & .026 & 0.605 \\
\hline Lengua extranjera & $0.305(0.084)$ & .195 & $3.628^{d}$ & $0.309(0.084)$ & .198 & $3.685^{d}$ & $0.229(0.080)$ & .146 & $2.859^{c}$ \\
\hline Matemáticas & $0.117(0.085)$ & .073 & 1.372 & $0.126(0.085)$ & .079 & 1.479 & $0.063(0.081)$ & .039 & 0.777 \\
\hline Ciencias sociales & $0.250(0.100)$ & .120 & $2.500^{c}$ & $0.251(0.100)$ & .120 & $2.514^{\mathrm{C}}$ & $0.205(0.095)$ & .098 & $2.158^{\mathrm{b}}$ \\
\hline Ciencias naturales & $0.306(0.105)$ & .138 & $2.930^{c}$ & $0.313(0.104)$ & .141 & $3.005^{c}$ & $0.287(0.099)$ & .129 & $2.902^{c}$ \\
\hline Educación artística & $-0.012(0.106)$ & -.005 & -0.110 & $-0.010(0.107)$ & -.005 & -0.094 & $-0.057(0.101)$ & -.026 & -0.571 \\
\hline Tecnología & $0.509(0.128)$ & .176 & $3.981^{\mathrm{d}}$ & $0.506(0.128)$ & .176 & $3.955^{d}$ & $0.255(0.123)$ & .088 & $2.064^{\mathrm{b}}$ \\
\hline Política sobre enseñanza con tecnología & & & & $0.153(0.077)$ & .085 & $1.986 b$ & $0.109(0.073)$ & .060 & 1.497 \\
\hline Política sobre gestión con tecnología & & & & $0.023(0.098)$ & .010 & 0.235 & $0.052(0.092)$ & .023 & 0.561 \\
\hline Infraestructura tecnológica & & & & $0.180(0.101)$ & .075 & $1.793^{\mathrm{a}}$ & $0.040(0.096)$ & .017 & 0.420 \\
\hline Ratio ordenadores alumno & & & & $-0.016(0.024)$ & -.026 & -0.645 & $-0.014(0.023)$ & -.023 & -0.598 \\
\hline \multicolumn{10}{|l|}{ Apoyo al uso de la tecnología } \\
\hline No & & & & - & - & - & - & - & - \\
\hline Sí & & & & $-0.018(0.054)$ & -.013 & -0.335 & $-0.062(0.051)$ & -.046 & -1.215 \\
\hline Alfabetización digital & & & & & & & $0.170(0.058)$ & .137 & $2.910^{c}$ \\
\hline \multicolumn{10}{|l|}{ Formación uso educativo tecnología } \\
\hline Ninguna & & & & & & & - & - & - \\
\hline Sí, poco útil & & & & & & & $0.002(0.069)$ & .001 & 0.025 \\
\hline Sí, muy útil & & & & & & & $0.248(0.058)$ & .191 & $4.281^{\mathrm{d}}$ \\
\hline \multicolumn{10}{|l|}{ Acceso a Internet en la escuela } \\
\hline Nunca o muy poco & & & & & & & - & - & - \\
\hline Mensual & & & & & & & $-0.070(0.094)$ & -.041 & -0.751 \\
\hline Semanal & & & & & & & $-0.015(0.090)$ & -.011 & -0.169 \\
\hline Diario & & & & & & & $0.148(0.090)$ & .113 & $1.650^{\mathrm{a}}$ \\
\hline \multicolumn{10}{|l|}{ Acceso a Internet fuera la escuela } \\
\hline Nunca o muy poco & & & & & & & - & - & - \\
\hline Mensual & & & & & & & $0.141(0.105)$ & .059 & 1.334 \\
\hline Semanal & & & & & & & $0.228(0.086)$ & .150 & $2.669^{c}$ \\
\hline Diario & & & & & & & $0.199(0.082)$ & .153 & $2.427^{b}$ \\
\hline \multicolumn{10}{|l|}{ Resumen del modelo } \\
\hline R2 (R2 ajustada) & \multicolumn{3}{|c|}{$.261(.046)$} & \multicolumn{3}{|c|}{$.286(.052)$} & \multicolumn{3}{|c|}{$.451(.165)$} \\
\hline F para el modelo & & $27^{c}$ & & & $2.744^{c}$ & & & $328^{c}$ & \\
\hline Cambio de R2 & & 68 & & & .014 & & & 21 & \\
\hline F para el cambio de R2 & & $27^{c}$ & & & 1.835 & & & $243^{c}$ & \\
\hline
\end{tabular}


Los datos proporcionados por el resumen de cada modelo nos permiten comprobar la influencia de las variables de cada categoría en la variable independiente. El modelo 1, compuesto por las variables de control que incluyen las características estructurales de la escuela y las características individuales del profesor, es estadísticamente significativo $(\mathrm{F}=3.027, \mathrm{p}<.01)$ y proporciona un grado aceptable de varianza explicada $\left(\mathrm{R}^{2}=.261\right)$. El modelo 2, que incorpora las variables referidas a las características tecnológicas de la escuela, se mantiene estadísticamente significativo $(\mathrm{F}=2.744, \mathrm{p}<.01)$ y ofrece una varianza explicada algo superior $\left(\mathrm{R}^{2}=.286\right)$, pero el cambio respecto al modelo 1 (F para el cambio de $\mathrm{R}^{2}=1.835$ ) no es significativo. Estos resultados justifican la conveniencia de progresar hacia un tercer modelo, que contemple los efectos del total de variables incluidas en el estudio y constituya, por tanto, el paso final del análisis de regresión jerárquica. Dicho modelo presenta un ajuste global aceptable $(\mathrm{F}=5.328, \mathrm{p}<.01)$ y muestra un cambio estadísticamente significativo ( $\mathrm{F}$ para el cambio de $\mathrm{R}^{2}=10.243, \mathrm{p}<.01$ ) respecto al modelo 2, con una mejora importante de la $\mathrm{R}^{2}$ a un valor de .451 .

De manera consistente con los efectos observados en el modelo 1, el modelo 2 muestra un efecto positivo significativo del género y de algunas áreas curriculares. Específicamente, ambos modelos indican que los hombres (Beta=.111, $\mathrm{p}<.01$ ) cuyas áreas curriculares son la lengua extranjera (Beta=.198, $\mathrm{p}<.001)$, la tecnología (Beta $=.176$, $\mathrm{p}<.001$ ), las ciencias naturales (Beta $=.141$, $\mathrm{p}<.01)$ y las ciencias sociales (Beta $=.120$, $\mathrm{p}<.01)$ tienen una mayor percepción de los beneficios educativos de los medios educativos digitales que sus homólogos. Asimismo, de manera coherente con el análisis bivariado, en ninguno de los dos modelos el nivel educativo y el número de habitantes son estadísticamente significativos. Por otra parte, en el modelo 2 se observan efectos negativos significativos de la edad (Beta=-.068, $\mathrm{p}<.10$ ) y de la titularidad privada de los centros (Beta=$.092, \mathrm{p}<.10)$ que no estaban presentes en el modelo 1. Por último, en relación a las variables sobre los aspectos institucionales de las escuelas introducidas en el modelo 2 y que no figuraban en el modelo 1 , se observa que, de todas ellas, únicamente la política sobre enseñanza con TIC (Beta $=.085, \mathrm{p}<.05)$ y la infraestructura (Beta $=.075, \mathrm{p}<.10)$ tienen un efecto significativo, en ambos casos positivo, sobre la percepción de los beneficios de los medios educativos digitales.

El modelo 3 muestra que, en relación a las variables de las características estructurales de la escuela y las características individuales del profesor, únicamente las áreas curriculares se mantienen significativas y tienen un efecto positivo en el caso de la lengua extranjera (Beta $=.146, \mathrm{p}<.01$ ), las ciencias naturales (Beta=.129, $\mathrm{p}<.01$ ), las ciencias sociales (Beta $=.098, \mathrm{p}<.05)$ y la tecnología (Beta $=.088$, $\mathrm{p}<.05)$. Sin embargo, a diferencia de los modelos anteriores, otras variables de control como el tipo de titularidad, el género y la edad dejan de contribuir de manera significativa a la explicación del modelo. Así mismo, ninguna de las variables relativas a las características tecnológicas de las escuelas predice de manera significativa la variable dependiente. Por lo tanto, a diferencia del modelo 2, factores como la política sobre enseñanza con TIC y la infraestructura dejan de ser significativos en el modelo 3. En último lugar, en cuanto a los efectos de las variables referentes a las características tecnológicas del profesor introducidas en el tercer paso, el hecho de tener niveles mayores de alfabetización digital (Beta=.137, $\mathrm{p}<.01$ ), haber recibido formación muy útil sobre el uso educativo de la tecnología (Beta=.191, $\mathrm{p}<.001)$, acceder diariamente (Beta $=.113, \mathrm{p}<.10)$ a Internet en la escuela y acceder semanal (Beta $=.150, \mathrm{p}<.01$ ) $\mathrm{y}$ diariamente (Beta=.153, $\mathrm{p}<.05)$ a Internet fuera de la escuela son factores asociados de manera positiva y significativa con la percepción de los beneficios educativos de los medios educativos digitales.

\section{Conclusiones}

Los resultados de esta investigación profundizan en el conocimiento disponible 
sobre los factores que influyen en la percepción de los profesores de educación primaria y educación secundaria de los beneficios del uso de los medios educativos digitales en la enseñanza y el aprendizaje. Los resultados muestran que las características estructurales de la escuela y las características individuales del profesor (modelo 1), consideradas de forma aislada, influyen significativamente en la percepción de los profesores. Además, cuando se añaden nuevas variables (modelo 2) referidas a las características tecnológicas de la escuela, el modelo continúa siendo significativo, a pesar de que no mejore sustancialmente el modelo 1. Sin embargo, el modelo final (modelo 3) muestra que, cuando se consideran todas las variables incluidas en el estudio, las características tecnológicas del profesor son las variables más influentes, se mejora los modelos anteriores de forma significativa, y además ofrece una varianza explicada significativa y mucho mayor.

El modelo 3 evidencia la influencia significativa de los factores relacionados con las características tecnológicas del profesor, tales como el grado de alfabetización digital, la formación en tecnología educativa y la frecuencia de acceso a Internet, dentro y fuera de la escuela. Los resultados también destacan la influencia del área curricular del profesor, especialmente de lengua extranjera, ciencias naturales, ciencias sociales y tecnología. Además, también muestran la nula influencia del resto de variables referidas a las características estructurales de la escuela y las características individuales del profesor, y a las características tecnológicas de la escuela.

En la misma dirección que Perrota (2013), también hemos identificado una influencia significativa y positiva de algunas variables de la institución escolar, como por ejemplo el área curricular del profesor. Sin embargo, nuestro estudio sostiene que los factores más influentes no forman parte de las características institucionales de la escuela, sino más bien están relacionados con las características tecnológicas del profesor.
Además, los resultados de nuestro estudio confirman algunos hallazgos y contradicen otros del trabajo de Inan y Lowther (2010). Ambas investigaciones coinciden en señalar que el factor alfabetización digital de los profesores es una de las variables con mayor influencia en la percepción de los profesores. Sin embargo, al contrario de los resultados de estos autores, no hemos hallado una influencia significativa de otros factores relacionados con las características tecnológicas de la escuela, tales como la ratio de ordenadores por alumno y la disponibilidad de soporte a la tecnología.

Estos resultados sugieren nuevas líneas futuras de investigación. Si los principales factores que influyen en la percepción del profesor de la utilidad de los medios educativos digitales están estrechamente relacionados con las características tecnológicas del profesor y el tipo contenido curricular, otras características individuales del profesor relacionadas con la tecnología podrían estar implicadas, tales como las creencias de los profesores sobre la enseñanza con ordenadores (Kordaki, 2013) o los enfoques de enseñanza (Rosário, Núñez, Valle, Paiva y Polydoro, 2012).

\section{Referencias}

Badia, A., Meneses, J. y Sigalés, C. (2013). Teachers' perceptions of factors affecting the educational use of ICT in technology-rich classrooms. Electronic Journal of Research in Educational Psychology 11(3), 787-808. DOI:

http://dx.doi.org/10.14204/ejrep.31.13053

Christensen, R. y Knezek, G. (2001). Instruments for assessing the impact of technology in education. Computers in the Schools, 18(2-3), 5-25.

Christensen, R. y Knezek, G. (2008). Selfreport measures and findings for information technology attitudes and competencies. En J. Voogt y G. Knezek (Eds.) International handbook of information technology in primary and secondary education (pp. 349365). New York: Springer. 
Inan, F. A. y Lowther, D. L. (2010). Factors affecting technology integration in $\mathrm{K}-12$ classrooms: a path model. Educational Technology Research and Development, 58(2), 137-154.

Knezek, G. A., Christensen, R. W. y Fluke, R. (2003, April). Testing a will, skill, tool model of technology integration. Paper presented at the American Educational Research Association (AERA), Chicago, IL.

Kordaki, M. (2013). High school computing teachers' beliefs and practices: A case study. Computers \& Education, 68, 141-152.

Morales, C. (2006). Cross-cultural validation of the will, skill, tool model of technology integration. Unpublished doctoral dissertation, University of North Texas, Denton, TX.

Mueller, J., Wood, E., Willoughby, T., Ross, C. y Specht, J. (2008). Identifying discriminating variables between teachers who fully integrate computers and teachers with limited integration. Computers \& Education, 51, 1523-1537.

Perrotta, C. (2013). Do school-level factors influence the educational benefits of digital technology? A critical analysis of teachers' perceptions. British Journal of Educational Technology, 44(2), 314-327.

Petko, D. (2012). Teachers' pedagogical beliefs and their use of digital media in classrooms: Sharpening the focus of the 'will, skill, tool' model and integrating teachers' constructivist orientations. Computers \& Education, 58(4), 1351-1359.

Rosario, P., Núñez, J. C., Valle, A., Paiva, O. y Polydoro, S. (2012). Approaches to Teaching in High School when Considering Contextual Variables and Teacher Variables//Enfoques de enseñanza en Bachillerato en función de variables contextuales y del docente. Revista de Psicodidáctica/Journal of Psychodidactics, 18(1).

Sigalés, C., Mominó, J. M., Meneses, J. y Badía, A. (2008). La integración de internet en la educación escolar española: situación actual y perspectivas de futuro. Barcelona: UOC. Retrieved from: http://www.fundacion.telefonica.com/debate yconocimiento/publicaciones/informe_escuel as/esp/pdf/informe_escuelas.pdf

Tondeur, J. (2007). Development and validation of a model of ICT integration in primary education. Tesis doctoral no publicada. Ghent University: Belgium.

Tondeur, J., Valcke, M. y Van Braak, J. (2008). A multidimensional approach to determinants of computer use in primary education: Teacher and school characteristics. Journal of Computer Assisted Learning, 24(6), 494-506.

Van Braak, J. (2001). Individual characteristics influencing teachers' class use of computers. Journal of Educational Computing Research, 25(2), 141-158.

Van Braak, J., Tondeur, J. y Valcke, M. (2004). Explaining different types of computer use among primary school teachers. European Journal of Psychology of Education, 19(4), 407-422.

Wastiau, P., Blamire, R., Kearney, C., Quittre, V., Van de Gaer, E. y Monseur, C. (2013). The Use of ICT in Education: a survey of schools in Europe. European Journal of Education, 48(1), 11-27.

\section{Agradecimientos}

Este artículo ha sido escrito en el marco del proyecto "La integración escolar de Internet en la educación escolar española. Situación actual $\mathrm{y}$ perspectiva de futuro (http://www.uoc.edu/in3/integracion_internet educacion_escolar/esp)", en el marco del Internet Interdisciplinary Institute (IN3), y con la financiación de la "Fundación Telefónica". También deseamos agradecer la ayuda recibida por parte de la Universitat Oberta de Catalunya para la traducción del artículo al inglés. 


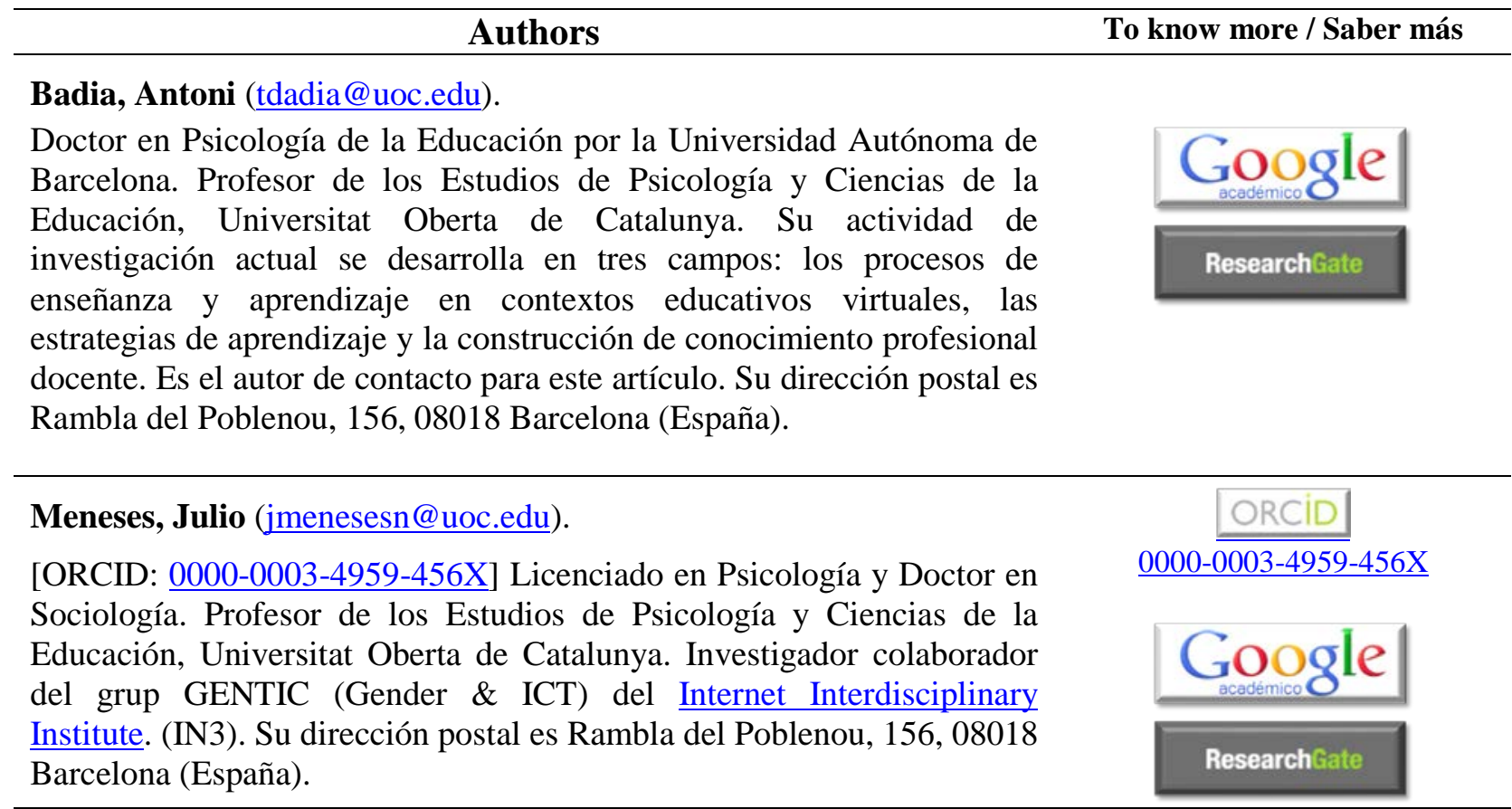

Fàbregues Sergi (sfabreguesf@uoc.edu).

Licenciado y Master en Sociología. Profesor de los Estudios de Psicología y Ciencias de la Educación, Universitat Oberta de Catalunya. Su dirección postal es Rambla del Poblenou, 156, 08018 Barcelona (España).

Sigalés, Carles (csigales@uoc.edu).

Profesor de Políticas Educativas, e investigador del Internet Interdisciplinary Institute (IN3), el Instituto de investigación de la Universitat Oberta de Catalunya (UOC). Actualmente está de Vicerrector de Política Académica y Profesorado en la UOC.

\section{RELIEVE}

\section{Revista ELectrónica de Investigación y $\mathbf{E V}$ aluación Educativa E-Journal of Educational Research, Assessment and Evaluation}

[ISSN: 1134-4032]

(C) Copyright, RELIEVE. Reproduction and distribution of this articles it is authorized if the content is no modified and their origin is indicated (RELIEVE Journal, volume, number and electronic address of the document).

(C) Copyright, RELIEVE. Se autoriza la reproducción y distribución de este artículo siempre que no se modifique el contenido y se indique su origen (RELIEVE, volumen, número y dirección electrónica del documento). 